\title{
The endomorphism type of certain bipartite graphs and a characterization of projective planes
}

\author{
Ralf Köhl (né Gramlich)* Katja Möser Hendrik Van Maldeghem ${ }^{\dagger}$
}

October 11, 2011

\begin{abstract}
In [2] Fan determines the endomorphism type of a finite projective plane. In this note we show that Fan's result actually characterizes the class of projective planes among the finite bipartite graphs of diameter three. In fact, this will follow from a generalization of Fan's theorem and its converse to all finite bipartite graphs with diameter $d$ and girth $g$ such that (1) $d+1<g \leq 2 d$, and (2) every pair of adjacent edges is contained in a circuit of length $g$.
\end{abstract}

\section{Introduction}

In this note, we generalize a theorem of Fan [2], obtaining the following characterization of bipartite graphs with diameter $d$ and girth $g$ such that $d+1<g \leq 2 d$ and such that every pair of adjacent edges is contained in a circuit of length $g$.

Main Result. Let $\Gamma$ be a finite bipartite graph with diameter $d$ and girth $g$ such that every pair of adjacent edges is contained in a circuit of length $g$. Then $d+1<g \leq 2 d$ if and only if the endomorphism type of $\Gamma$ is 6 if and only if this endomorphism type is even.

This implies in particular the following rather unexpected characterization of finite (generalized) projective planes in terms of the endomorphism type of their incidence graph.

Main Corollary. Let $\Gamma$ be a finite bipartite graph with diameter 3 . Then $\Gamma$ is a weak generalized triangle if and only if its endomorphism type is 6 if and only if its endomorphism type is even.

In Section 2 of this note we remind the reader of the definition of the endomorphism type of a finite graph as introduced in [1]. Section 3 contains the proof of the "only if" part of our Main Result (a direct generalization of Fan's result [2]). Finally, in Section 4 we conclude the proof of our Main Result by showing the "if" part. This article is based on the second author's Diplomarbeit [4].

\section{Endomorphism types}

In [1] the concept of the endomorphism type of a finite graph has been introduced. By [2] the endomorphism type of a finite generalized triangle is known. The purpose of this note is to characterize finite generalized triangles by their endomorphism type. Before doing so we recall the notions from [1].

Let $\Gamma=(V, E)$ be a finite undirected unweighted graph without loops and multiple edges. An endomorphism of $\Gamma$ is a map $V \rightarrow V$ which preserves edges. Since $\Gamma$ does not have loops

\footnotetext{
*The first author gratefully acknowledges the Heisenberg fellowship of the Deutsche Forschungsgemeinschaft during which this note has been written.

†The third author is partly supported by a Research Grant of the Fund for Scientific Research - Flanders (FWO - Vlaanderen).
} 
this in particular means that two adjacent vertices cannot have the same image under an endomorphism. An endomorphism $f$ is called halfstrong if each edge $\left(f\left(x_{1}\right), f\left(x_{2}\right)\right) \in E$ in the image of $f$ admits an edge in its preimage, i.e., there exists an edge $\left(y_{1}, y_{2}\right) \in E$ satisfying $\left(f\left(y_{1}\right), f\left(y_{2}\right)\right)=\left(f\left(x_{1}\right), f\left(x_{2}\right)\right)$. An endomorphism $f$ is called locally strong if for each edge $\left(f\left(x_{1}\right), f\left(x_{2}\right)\right) \in E$ the preimage $f^{-1}\left(f\left(x_{1}\right), f\left(x_{2}\right)\right)$ does not admit isolated vertices. An endomorphism $f$ is called quasistrong if for each edge $\left(f\left(x_{1}\right), f\left(x_{2}\right)\right) \in E$ in the image of $f$ there exist preimages $y_{1} \in f^{-1}\left(f\left(x_{1}\right)\right)$ adjacent to each preimage of $f\left(x_{2}\right)$ and $y_{2} \in f^{-1}\left(f\left(x_{2}\right)\right)$ adjacent to each preimage of $f\left(x_{1}\right)$. An endomorphism $f$ is called strong if for each edge $\left(f\left(x_{1}\right), f\left(x_{2}\right)\right) \in E$ in the image of $f$ each preimage of $f\left(x_{1}\right)$ is adjacent to each preimage of $f\left(x_{2}\right)$. Finally, an endomorphism $f$ of $\Gamma$ is called an automorphism if it is bijective and its inverse map is also an endomorphism. Since $\Gamma$ is finite, an endomorphism is an automorphism if and only if it is injective.

The set of endomorphisms, halfstrong endomorphisms, locally strong endomorphisms, quasistrong endomorphisms, strong endomorphisms, and automorphisms of a finite graph $\Gamma$ is denoted by $\operatorname{End}(\Gamma), \operatorname{HEnd}(\Gamma)$, LEnd $(\Gamma), Q \operatorname{End}(\Gamma), \operatorname{SEnd}(\Gamma), \operatorname{Aut}(\Gamma)$, respectively. By definition

$$
\operatorname{Aut}(\Gamma) \subseteq \operatorname{SEnd}(\Gamma) \subseteq \operatorname{QEnd}(\Gamma) \subseteq \operatorname{LEnd}(\Gamma) \subseteq \operatorname{HEnd}(\Gamma) \subseteq \operatorname{End}(\Gamma)
$$

To a graph one can associate a string of length five consisting of 0 s and 1 s indicating whether the corresponding inclusion is proper (1) or not (0). This string interpreted as a binary number is called the endomorphism type of the graph $\Gamma$. For instance, to a graph satisfying Aut $(\Gamma)=$ $\operatorname{SEnd}(\Gamma)=\mathrm{QEnd}(\Gamma) \subsetneq \operatorname{LEnd}(\Gamma) \subsetneq \operatorname{HEnd}(\Gamma)=\operatorname{End}(\Gamma)$ one associates the string 00110 which yields the endomorphism type 6 (written as a decimal number).

\section{The endomorphism type of certain bipartite graphs}

Bipartite graphs are the incidence graphs of point-line geometries. In fact, these geometries can be identified with their incidence graph and hence the study of point-line geometries is equivalent to the study of bipartite graphs. From this point of view, a weak generalized triangle, of in more common words, a generalized projective plane, is a bipartite graph with diameter 3 and girth 6 .

Fan [2] proved that a weak finite generalized triangle has endomorphism type 6 . Inspection shows that his proof can be extended to arbitrary bipartite graphs with diameter $d$ and girth $g$ satisfying $d+1<g \leq 2 d$ and such that every pair of adjacent edges is contained in a circuit of length $g$. We adapt his proof here to this class of bipartite graphs for the reader's convenience. Note that the condition that every pair of adjacent edges is contained in a circuit of length $g$ is not a very restrictive one. Indeed, on the one hand most geometries satisfy this condition, on the other hand the most important application of point-line geometries is in permutation groups, where a "sufficiently transitive" automorphism group easily implies that condition.

Theorem 3.1 Let $d \geq 3$ and let $\Gamma$ be a finite bipartite graph with diameter $d$ and girth $g$, with $d+1<g \leq 2 d$ and such that every pair of adjacent edges is contained in a circuit of length $g$. Then the endomorphism type of $\Gamma$ equals 6.

Proof. We split the proof into four parts.

Lemma 3.2 $\operatorname{End}(\Gamma)=\operatorname{HEnd}(\Gamma)$.

By way of contradiction assume the existence of an endomorphism $f$ which is not halfstrong. Then there exists an edge $\left(f\left(x_{1}\right), f\left(x_{2}\right)\right) \in E$ with the property that no preimage of $f\left(x_{1}\right)$ is adjacent to any preimage of $f\left(x_{2}\right)$. In particular, $x_{1}$ and $x_{2}$ are not adjacent. There exists a path $x_{1}$, $v_{1}, v_{2}, \ldots, v_{k-1}, x_{2}$ from $x_{1}$ to $x_{2}$. Since $v_{1}$ is adjacent to $x_{1}$ we have $f\left(v_{1}\right) \neq f\left(x_{1}\right)$. Similarly, $f\left(v_{k-1}\right) \neq f\left(x_{2}\right)$. Therefore the path $f\left(x_{1}\right), f\left(v_{1}\right), f\left(v_{2}\right), \ldots, f\left(v_{k-1}\right), f\left(x_{2}\right)$ contains at least four distinct elements, yielding a circuit of length at most $d+1$. This contradicts the assumption on the girth $g$.

Lemma 3.3 $\operatorname{HEnd}(\Gamma) \neq \operatorname{LEnd}(\Gamma)$. 
By definition of diameter, there is a vertex $x_{0}$ of $\Gamma$ such that each of the sets $\Gamma_{i}\left(x_{0}\right):=\{x \in$ $\left.\Gamma \mid \operatorname{dist}_{\Gamma}\left(x_{0}, x\right)=i\right\}, 0 \leq i \leq d$ is non-empty. Fix elements $x_{1} \in \Gamma_{1}\left(x_{0}\right)$ and $x_{2} \in \Gamma_{2}\left(x_{0}\right)$ with $\left(x_{1}, x_{2}\right) \in E$. Define an endomorphism $f$ via

$$
f(x)= \begin{cases}x_{0} & \text { if } x=x_{0}, \\ x_{1} & \text { if } x \in \Gamma_{2 k-1}\left(x_{0}\right), k \in \mathbb{N}, \\ x_{2} & \text { if } x \in \Gamma_{2 k}\left(x_{0}\right), k \in \mathbb{N} .\end{cases}
$$

This endomorphism is halfstrong but not locally strong. Indeed, let $y \in \Gamma_{3}\left(x_{0}\right)$. Then $f(y)=x_{1}$ and $y$ is an isolated vertex in the preimage $f^{-1}\left(\left\{x_{0}, x_{1}\right\}\right)$, because $y$ is not adjacent to $x_{0}$ (it has distance 3 from $\left.x_{0}\right)$ and not adjacent to any vertex in $f^{-1}\left(x_{1}\right)=\bigcup_{k} \Gamma_{2 k-1}\left(x_{0}\right)$ (because the graph $\Gamma$ is bipartite).

Lemma 3.4 LEnd $(\Gamma) \neq \operatorname{QEnd}(\Gamma)$.

As before let $x_{0}$ be a vertex of $\Gamma$ such that $\Gamma_{d}(x) \neq \emptyset$ and fix elements $x_{1} \in \Gamma_{1}\left(x_{0}\right)$ and $x_{2} \in \Gamma_{2}\left(x_{0}\right)$ with $\left(x_{1}, x_{2}\right) \in E$. Define an endomorphism $f$ via

$$
f(x)= \begin{cases}x_{1} & \text { if } x \in \Gamma_{2 k+1}\left(x_{0}\right), k \in \mathbb{N} \cup\{0\}, \\ x_{2} & \text { if } x \in \Gamma_{2 k}\left(x_{0}\right), k \in \mathbb{N} \cup\{0\} .\end{cases}
$$

This endomorphism is locally strong but not quasistrong.

Lemma 3.5 QEnd $(\Gamma)=\operatorname{Aut}(\Gamma)$.

Assume that $f$ is a quasistrong endomorphism which is not an automorphism. This means that $f$ is not injective, so there exist vertices $x_{1}, x_{2}$ with $f\left(x_{1}\right)=f\left(x_{2}\right)$. Since edges cannot collapse, the vertices $x_{1}$ and $x_{2}$ are not adjacent. On the other hand, since $f$ is quasistrong, the vertices $x_{1}$ and $x_{2}$ necessarily have a common neighbor. Therefore $x_{1}$ and $x_{2}$ have distance 2 in $\Gamma$. Now, however, any circuit of length $g$ of $\Gamma$ containing $x_{1}$ and $x_{2}$ (and by assumption there exists at least one such) is collapsed by $f$ to a circuit of insufficient length, a contradiction.

Remark 3.6 In Theorem 3.1 we cannot dispense with the bipartite assumption. Indeed, the endomorphism type of the graph with vertex set all 3-subsets of a set of 7 elements, and adjacency being disjoint, is 0 as the reader can check for himself. The graph has diameter 3 and girth 6 .

Remark 3.7 The endomorphism type of a complete bipartite graph (the case $d=2$ of the previous theorem) is 16 , cf. [4].

\section{Bipartite graphs with even endomorphism type}

Theorem 4.1 Let $d \geq 2$ and let $\Gamma$ be a bipartite graph with diameter $d$ and finite girth, and with even endomorphism type. Then $n+1<g \leq 2 n$, where $g$ denotes the girth of $\Gamma$.

Proof. First note that, if $d=2$, then $g=4$ and we are done. Now suppose $d \geq 3$.

Since $\Gamma$ is bipartite, its girth $g$ is even, whence $g \leq 2 d$. We have to prove that, if $d+1 \geq g$, then a non-halfstrong endomorphism exists. Since the girth is $g$ there exists a circuit $y_{0}, y_{1}, \ldots$, $y_{g-1}, y_{g}=y_{0}$. We assume by way of contradiction that $g \leq d+1$. Define a map $f$ via

$$
f(x)= \begin{cases}y_{i} & \text { if } x \in \Gamma_{i}\left(y_{0}\right) \text { and } i \leq g-1, \\ y_{g-2} & \text { if } x \in \Gamma_{g+2 i}\left(y_{0}\right) \text { for some } i \geq 0, \\ y_{g-1} & \text { if } x \in \Gamma_{g-1+2 i}\left(y_{0}\right) \text { for some } i \geq 0 .\end{cases}
$$

Note that the map $f$ does not collapse edges, because every circuit has even length, so that $f$ is an endomorphism. Since $g \leq d+1$ the vertex $y_{g-1}$ is contained in the image of $f$. Hence the edge 
$\left(y_{0}, y_{g-1}\right)$ is in the image, but clearly has no edge in its pre-image. Therefore the endomorphism $f$ is not halfstrong, a contradiction.

The Main Result follows from Theorem 3.1 and Theorem 4.1. Also, the Main Corollary follows from the Main result by noting that, in a generalized projective plane, every pair of adjacent edges is contained in a circuit of length 6 .

Remark 4.2 Also in Theorem 4.1 we cannot dispense with the bipartite assumption. Indeed, consider a (finite) graph $\Gamma$ with diameter 4 and girth 8 (a so-called generalized quadrangle, see [5]). Now let $\Gamma^{\prime}$ be the adjacency graph of a generalized quadrangle, i.e., the vertex set of $\Gamma^{\prime}$ is one of the partitions of $\Gamma$ and two vertices in $\Gamma^{\prime}$ are adjacent if they are at distance 2 in $\Gamma$. Note that this graph has diameter two and girth three, and therefore does not satisfy the conclusion of Theorem 4.1. The endomorphism $f$ induces an endomorphism $\phi$ of the incidence graph of the generalized quadrangle which is bijective on the point rows. Theorem 5.1 of [3] implies that $\phi$ and hence $f$ is either an automorphism or the image of $f$ consists of a clique. In both cases $f$ is halfstrong, whence the endomorphism type is even.

Remark 4.3 The prominent examples of bipartite graphs with large girth compared to the diameter are the so-called generalized polygons, which have diameter $d$ and girth $2 d$, see [5]. For these geometries, the condition that every pair of adjacent edges is contained in a circuit of length $g$ is automatically satisfied and hence our Main Result applies.

\section{References}

[1] M. Böttcher, U. Knauer, Endomorphism spectra of graphs, Discr. Math. 109 (1992), 45-57.

[2] S. Fan, Endomorphism spectra of bipartite graphs with diameter three and girth six, Southeast Asian Bull. Math. 25 (2001), 217-221.

[3] R. Gramlich, H. Van Maldeghem, Epimorphisms of generalized polygons. Part 1: geometrical characterizations, Des. Codes Cryptogr. 21 (2000), 99-111.

[4] K. Möser, Endomorphismen von verallgemeinerten Vielecken, Diplomarbeit, TU Darmstadt 2007.

[5] H. Van Maldeghem, Generalized Polygons, Birkhäuser, Basel 1998.

Author's addresses

Ralf Köhl, Katja Möser

JLU Gießen

Mathematisches Institut

Arndtstraße 2

35392 Gießen

Germany

ralf.koehl@math.uni-giessen.de

$\mathrm{k}$.moeser@gmx.net

Hendrik Van Maldeghem

Ghent University

Pure Mathematics and Computer Algebra

Krijgslaan 281, S22

9000 Gent

Belgium

hvm@cage.ugent.be 